\title{
Non-linear separation of pressure, velocity and wave intensity into forward and backward components
}

\author{
Jonathan P. Mynard ${ }^{1,2}$ \\ Malcolm R. Davidson ${ }^{3}$ \\ Daniel J. Penny ${ }^{1,2}$ \\ Joseph J. Smolich ${ }^{1,2}$
}

${ }^{1}$ Heart Research Group, Murdoch Childrens Research Institute, ${ }^{2}$ Department of Paediatrics and ${ }^{3}$ Department of Chemical and Biomolecular Engineering, University of Melbourne, Melbourne, Australia

\section{Corresponding Author:}

Jonathan P. Mynard

E-mail: jonathan.mynard@mcri.edu.au

(Current address)

Biomedical Simulation Laboratory, University of Toronto

5 King's College Rd, Toronto, Ontario, Canada, M5S 3G8

Phone: +1 4169787773

Fax: $\quad+14169787753$

Word count:

Abstract: 195

Total: 3000 


\begin{abstract}
Separating pressure, flow/velocity and wave intensity signals into forward and backward components provides insights about arterial wave propagation and reflection. A linear wave separation is normally used, but ignores the pressure-dependence of wave speed. While a nonlinear separation could incorporate this pressure-dependence, no such method exists for wave intensity decomposition. Moreover, although linear separation errors for pressure $(5-10 \%)$ have been quantified previously, errors for velocity and wave intensity have not. Accordingly, we describe a non-linear wave separation technique based on the method of characteristics. Data from a computer model suggest that the percentage linear separation errors for velocity and wave intensity are approximately one-half and twice that for pressure respectively. Although comparable to measurement uncertainty in many instances, linear separation errors may become more significant 1) if wave speed varies substantially over the cardiac cycle, e.g. if pulse pressure or vessel compliance is high, 2) if the degree of wave reflection in the arterial system is large, or 3) if the constant wave speed used for the linear separation is closer to the minimum or maximum pressure-dependent value rather than the mean. Consideration of linear separation errors may therefore be important in some physiological settings.
\end{abstract}

Keywords: wave intensity analysis, arterial haemodynamics, wave separation, wave speed 


\section{Introduction}

In studies of arterial haemodynamics, understanding how upstream and downstream phenomena contribute to measured signals is crucial. Hence, 'wave separation' is used to decompose a pressure, flow/velocity or wave intensity signal into calculated waveforms related to forward-running and backward-running waves, thus permitting elucidation of haemodynamic interactions between the ventricle and vasculature [2] and between different vascular regions [18].

Wave separation can be achieved in the frequency-domain [21] or time-domain [7, 8], and to date has generally assumed linearity, i.e. forward and backward waves are additive and local wave speed is constant throughout the cardiac cycle $[12,14,21]$. However, wave speed is known to be pressure-dependent, with intra-beat variation of $20-30 \%$ [5] or substantially more in patients with hypertension [4]. This pressure-dependence means that the sum of the forward and backward components does not necessarily equal the net (i.e. measured) quantity. Several studies have investigated the effects of mild non-linearities on pressure decomposition $[13-15,20]$ and concluded that, under normal physiological conditions, errors involved in the linear approach are comparable to measurement uncertainty (i.e. $5-10 \%$ ). However, errors arising from linear separation of flow/velocity and wave intensity, and from substantial intra-cycle wave speed variations, have not been investigated, in part because a non-linear method for separating wave intensity into components has not yet been described. In this study, we present such a method and quantify linear separation errors for pressure, velocity and wave intensity. Linear and non-linear techniques are assessed with a simple onedimensional (1D) computer model in which the pressure-wave speed relation is prescribed, and in which key physiological variables likely to affect the linear separation errors (wave speed, wave reflection and pulse amplitude) are precisely controlled. 


\section{Methods}

The 1D equations governing pressure $(p)$, velocity $(u)$ and cross-sectional area $(A)$ in a compliant tube can be expressed as

$$
\begin{gathered}
\frac{\partial A}{\partial t}+\frac{\partial A u}{\partial x}=\psi \\
\frac{\partial u}{\partial t}+u \frac{\partial u}{\partial x}+\frac{1}{\rho} \frac{\partial p}{\partial x}=f
\end{gathered}
$$

where $\rho$ is blood density $\left(1.06 \mathrm{~g} / \mathrm{cm}^{3}\right), \psi$ represents fluid loss due to wall permeability and $f$ is a loss/source term arising from viscous friction and body forces. These equations can be recast [17] in terms of Riemann variables $\left(w_{ \pm}\right)$,

$$
\frac{\partial w_{ \pm}}{\partial t}+\lambda_{ \pm} \frac{\partial w_{ \pm}}{\partial x}=S
$$

where $S$ incorporates loss/source terms, $\lambda_{ \pm}=u \pm c(p)$ and $c(p)$ is wave speed. If $S$ is small, $w_{ \pm}$are related to $p$ and $u$ via

$$
w_{ \pm}=u \pm \int_{p_{0}}^{p} \frac{1}{\rho c\left(p^{\prime}\right)} d p^{\prime}
$$

where $p_{0}$ is a reference pressure. Parker and Jones [12] defined a 'wavelet' as an infinitesimal change in $w_{+}$or $w_{-}$,

$$
d w_{ \pm}=d u \pm \frac{1}{\rho c(p)} d p
$$

These wavelets propagate upstream or downstream at a speed given by $\lambda_{ \pm}$. Defining pressure and velocity changes associated with $d w_{ \pm}$in the absence of changes in $d w_{-}$as 'forward' components (i.e. $d p_{+}=\left.d p\right|_{d w_{-}=0}, d u_{+}=\left.d u\right|_{d w_{-}=0}$ ), and vice versa for 'backward' components $\left(d p_{-}=\left.d p\right|_{d w_{+}=0}, d u_{-}=\left.d u\right|_{d w_{+}=0}\right)$, from $(5)$ 


$$
d p_{ \pm}= \pm \rho c_{ \pm} d u_{ \pm}
$$

which is the well-known water-hammer equation revealing the coupled nature of pressure and velocity perturbations. Note that a constant wave speed is commonly used in (6) but here wave speed is expressed in component form $\left(c_{ \pm}\right)$, recognising that this is a pressuredependent variable that would differ from net $c$ if only forward or only backward waves existed. Wave intensity (defined as $d p d u$ ) is related to $w_{ \pm}$and $c$ via [12]

$$
d p d u=\frac{1}{4} \rho c\left(d w_{+}^{2}-d w_{-}^{2}\right)
$$

Hence, $d p d u>0$ when forward waves predominate and $d p d u<0$ when backward waves predominate. However, $d p d u$ only represents the net effect of forward and backward waves and thus a backward wave may not be detected if a larger forward wave exists around the same time. Similarly, the independent contribution of forward and backward waves to pressure and velocity waveforms is not immediately clear when viewing the raw signals. This problem necessitates wave separation techniques.

\subsection{Linear wave separation}

In experimental and clinical settings, the precise pressure-wave speed relation is often unknown, making evaluation of (4) impossible. Nevertheless, if a constant wave speed $(\bar{c})$ is assumed, and forward and backward components of pressure $\left(d p_{ \pm}\right)$and velocity $\left(d u_{ \pm}\right)$are additive, i.e. $d p=d p_{+}+d p_{-}$and $d u=d u_{+}+d u_{-}$, from (6)

$$
\begin{gathered}
d p_{ \pm}=\frac{1}{2}(d p \pm \rho \bar{c} d u) \\
d u_{ \pm}=\frac{1}{2}\left(d u \pm \frac{1}{\rho \bar{c}} d p\right)
\end{gathered}
$$

Multiplying (8) and (9) gives the corresponding components of wave intensity 


$$
d p_{ \pm} d u_{ \pm}= \pm \frac{1}{4 \rho \bar{c}}(d p \pm \rho \bar{c} d u)^{2}
$$

or in the commonly-used time-corrected form [16]:

$$
w i_{ \pm}= \pm \frac{1}{4 \rho \bar{c}}\left(\frac{d p}{d t} \pm \rho \bar{c} \frac{d u}{d t}\right)^{2}
$$

\subsection{Non-linear wave separation}

If the vessel pressure-wave speed relation is known, e.g. via the Bramwell-Hill equation [4], wave separation can be achieved without any linearising assumptions. Taking $d w_{-}=0$ (forward-running wavelets only, $d p=d p_{+}$and $d u=d u_{-}$) or $d w_{+}=0$ (backward-running wavelets only, $d p=d p_{-}$and $d u=d u_{-}$), evaluation of $d w_{+}$or $d w_{-}$respectively in (5) yields

$$
d w_{ \pm}=d u_{ \pm} \pm \frac{1}{\rho c_{ \pm}} d p_{ \pm}
$$

Substituting (6) into (12) allows calculation of $d p_{ \pm}$and $d u_{ \pm}$from $d w_{ \pm}$,

$$
\begin{gathered}
d p_{ \pm}= \pm \frac{\rho c_{ \pm}}{2} d w_{ \pm} \\
d u_{ \pm}=\frac{1}{2} d w_{ \pm}
\end{gathered}
$$

A non-linear separation of wave intensity can now be introduced by multiplying (13) and (14)

$$
d p_{ \pm} d u_{ \pm}= \pm \frac{\rho c_{ \pm}}{4} d w_{ \pm}^{2}
$$

or in time-corrected form,

$$
w i_{ \pm}= \pm \frac{\rho c_{ \pm}}{4}\left(\frac{d w_{ \pm}}{d t}\right)^{2}
$$

Importantly, since defining $d p_{ \pm}, d u_{ \pm}$and $w i_{ \pm}$on the basis of Riemann variables is a nonlinear approach, it is generally not the case that components are additive [14]. 


\subsection{Pressure and velocity components}

The cumulative effect of many infinitesimal wavelets on pressure and velocity over an arbitrary time can be determined by integrating the linear or non-linear expressions for $d p_{ \pm}$ and $d u_{ \pm}$

$$
\begin{aligned}
& p_{ \pm}(t)=p_{ \pm}^{0}+\int_{t_{0}}^{t} d p_{ \pm}\left(t^{\prime}\right) \\
& u_{ \pm}(t)=u_{ \pm}^{0}+\int_{t_{0}}^{t} d u_{ \pm}\left(t^{\prime}\right)
\end{aligned}
$$

where $p_{ \pm}^{0}$ and $u_{ \pm}^{0}$ are arbitrary initial values of $p_{ \pm}$and $u_{ \pm}$, here taken to be $p_{0}$ and zero respectively.

\subsection{Computer modelling}

We compare linear and non-linear separation techniques in a simple computer model. Details of the formulation and numerical solution have been described previously $[10,11]$. The model (Fig. 1a) consists of a single $1 \mathrm{D}$ vessel (length, $l=20 \mathrm{~cm}$, reference cross-sectional area, $A_{0}=4 \mathrm{~cm}$, reference wave speed, $c_{0}=4 \mathrm{~m} / \mathrm{s}$ ), a forward component of pressure prescribed at the inlet, and an outlet reflection coefficient of 0.5 unless otherwise stated. Note that since the inlet absorbs all backward waves and since the outlet is purely resistive, the model does not produce the exponential decay of diastolic pressure; however this phenomenon is not important for the purposes of this study. A physiologically-relevant pressure-area relation [10] for the $1 \mathrm{D}$ segment is enforced,

$$
p-p_{\mathrm{ext}}=\frac{2 \rho c_{0}^{2}}{b}\left[\left(\frac{A}{A_{0}}\right)^{\frac{b}{2}}-1\right]+p_{0}
$$

with external pressure $\left(p_{\text {ext }}\right)$ assumed to be zero, $p_{0}=p\left(A_{0}\right)$ as the reference pressure, and the constant $b$ controlling the pressure-dependence of wave speed as follows 


$$
c=\sqrt{\frac{b}{2 \rho}\left(p-p_{0}\right)+c_{0}^{2}}
$$

To adjust the degree of wave speed pressure-dependence, we set $b$ to low, intermediate and high values $(b=0.1,2.8$ and 8.0), resulting in pressure-area and pressure-wave speed relations shown in Fig. 1b\&c. For linear separation, the wave speed constant $\bar{c}$ is chosen to be the cycle-averaged value from (20) unless otherwise stated; we also investigate $\bar{c}=\max (c)$ and $\bar{c}=\min (c)$, noting that foot-to-foot [3] and $p u$-loop methods [6] most commonly used to calculate wave speed probably produce values closer to $\min (c)$.

\section{Results and Discussion}

Fig. 2 shows net $c, p$ and $u$ waveforms and the respective forward and backward components using linear and non-linear separation techniques. As with the left ventricle ejecting into the aorta, the input $p_{+}$produces a forward compression wave (FCW) that increases $p$ and $u$, followed by a forward expansion wave (FEW) that decreases $p$ and $u$. These waves propagate along the vessel and are partially reflected at the terminal, producing backward compression (BCW) and expansion (BEW) waves respectively. Note that although the two forward waves are the same size at the inlet and the terminal reflection coefficient is constant, the backward waves do not have the same amplitude because nonlinear propagation effects amplify the $\mathrm{FCW}$ and $\mathrm{BCW}$ and attenuate the $\mathrm{FEW}$ and $\mathrm{BEW}$ [9]. This effect also occurs when $b=0.1$, because although wave speed is constant, wavelets propagate along the non-linear paths $d x / d t=u \pm c$ and thus fully linear flow only occurs when changes in $u$ are negligibly small [9] or in a linearised formulation in which $d w_{ \pm}$ propagate along the linear paths $d x / d t= \pm c$ [1] 


\subsection{Linear vs.non-linear wave separation}

Differences between linear and non-linear separation techniques are negligible when wave speed is quasi-constant throughout the cardiac cycle $(b=0.1)$ and increase as wave speed becomes more pressure-dependent (Fig. 2). With $b=2.8$ (20\% wave speed variation), linear separation overestimates peak $p_{+}$and $p_{-}$by up to $6.5 \%$ of the signal amplitude, with greater errors in the backward component. For a highly pressure-dependent wave speed $(b=8.0$, $50 \%$ wave speed variation), the error in $p_{ \pm}$becomes more substantial (up to $16 \%$ ). In both instances, maximum $p_{ \pm}$errors occur around the time of peak pressure. Linear separation of $u$ causes smaller errors than for $p$, with minor underestimation of $u_{+}$(peak errors $-2 \%$ and $-4 \%$ for $b=2.8$ and $b=8.0$ respectively) and overestimation of $u_{-}(3 \%$ and $7 \%)$. Unlike $p_{ \pm}$errors, the greatest $u_{ \pm}$errors occur during acceleration and deceleration. Maximal errors for pressure and velocity separation thus appear to lie within the range of measurement uncertainty, as concluded previously $[13-15,20]$, except for the pressure signal under substantially non-linear conditions.

The non-linear separation correctly predicts that peak wave intensity for the FCW and FEW are equal (noting that the input $p_{+}$is symmetric), whereas the linear method overestimates FEW amplitude by $6 \%(b=2.8)$ and $15 \%(b=8.0)$ since it is calculated from net $p, u$ and $c$ which are all affected by the BCW. Similarly, BCW amplitude is overestimated (by $12 \%$ and $32 \%$ ) because of the pressure effects of the preceding FCW. Note that such wi errors, although greater than corresponding $p$ and $u$ errors, may still be comparable with measurement errors in physiological studies, given that wi is calculated from the product of $p$ and $u$ differentials, and is therefore more sensitive to noise and movement artefact. 
Considering $b=8$, if minimum or maximum intra-beat wave speed are used for the linear separation, maximum absolute $p_{-}$and $u_{-}$errors $(19-28 \%)$ are greater than if mean wave speed is used $(5-16 \%)$ and the time course of the error is altered substantially (Fig. 3). By contrast, errors in $\mathrm{BCW}$ and $\mathrm{FEW}$ peak wi are relatively unaffected by the choice of $\min /$ mean/max wave speed. In all cases, the linear separation introduces small artefactual backward waves beneath the two forward waves which, although negligible in some instances, have appreciable pressure/velocity effects when using minimum or maximum wave speed values (see arrows in Fig. 3).

Fig. 4 shows the dependence of maximum linear separation errors (using mean wave speed) on key model parameters. Reducing reference wave speed $\left(c_{0}\right)$ causes exponential rises in the errors of all $u, p$ and wi components (all $R^{2}>0.97$ ); in the most non-linear case tested $\left(b=8, c_{0}=2 \mathrm{~m} / \mathrm{s}\right)$, errors in the backward components are substantial $(16 \%, 41 \%$ and $83 \%$ respectively). Importantly, decreasing $c_{0}$ also leads to an increase in the intra-beat percentage variation of wave speed ( 7 to $67 \%$ for $b=2.8$ and 18 to $156 \%$ for $b=8$ over the range of $c_{0}$ tested). All errors exhibit linear dependence on the input pulse amplitude $\left(R^{2}>0.98\right)$, again most likely due to a pressure-dependent increase in wave speed variation. Varying the terminal reflection coefficient between -1 and +1 has a relatively minor effect on the percentage error of all backward components, while the maximal $p_{+}, u_{+}$and $w i_{+}$errors display a linear relation with reflection coefficient $\left(R^{2}>0.98\right)$. Finally, with all other parameters fixed, increasing or decreasing $A_{0}$ by $50 \%$ has no effect on linear separation errors (results not shown). 


\subsection{Perspectives and limitations}

The foregoing results suggest that while linear wave separation is probably acceptable in most circumstances, non-linear effects may introduce substantial error in some situations. For example, non-linear effects are likely to be important in the fetal pulmonary circulation, since pulmonary arteries are highly compliant (wave speeds of 2.5-3.0 m/s [18]), there is a very large mid-systolic backward-running compression wave [18] and pulse pressure is moderate at baseline but substantial during ductal constriction [19]. Non-linear effects may also be important in adults with high systolic blood pressure, in whom large differences between systolic and diastolic wave speed have been reported [4].

Friction and wall viscoelasticity were not addressed in this study and require further investigation. In settings where wave patterns differ substantially from those produced by our simple model, the magnitude and time course of linear separation errors may also differ.

\subsection{Conclusions}

We described a non-linear technique for separating wave intensity into forward and backward components. Data from a 1D model indicated that under many normal physiological conditions, linear wave separation is likely to cause errors of $2-5 \%$ for velocity, $5-10 \%$ for pressure and $10-20 \%$ for wave intensity. Although such errors may be comparable with measurement uncertainty, substantially greater errors may arise if minimum or maximum wave speed is used rather than the mean, if wave speed varies substantially during the cardiac cycle or if there is a large degree of wave reflection in the arterial network. Linear separation may also introduce small artefactual backward waves. Given a pressure-area relation, these errors can be avoided by performing non-linear wave separation. 


\section{Acknowledgments}

This work was supported by a National Heart Foundation of Australia Grant-in-Aid, the Victorian Government Operational Infrastructure Support Program and a National Health and Medical Research Council of Australia Dora Lush Biomedical Scholarship (J. P. Mynard).

\section{References}

[1] Alastruey J, Parker K, Peiró J, and Sherwin S (2009) Analysing the pattern of pulse waves in arterial networks: a time-domain study. J Eng Math 64:331-351.

[2] Chirinos JA and Segers P (2010) Noninvasive evaluation of left ventricular afterload: Part 2: Arterial pressure-flow and pressure-volume relations in humans. Hypertension $56: 563-570$.

[3] Hamilton WF, Remington JW, and Dow P (1945) The determination of the propagation velocity of the arterial pulse wave. Am J Physiol 144:521-535.

[4] Hermeling E, Hoeks APG, Winkens MHM, Waltenberger JL, Reneman RS, Kroon AA, and Reesink KD (2010) Noninvasive assessment of arterial stiffness should discriminate between systolic and diastolic pressure ranges. Hypertension 55:124-130.

[5] Histand MB and Anliker M (1973) Influence of flow and pressure on wave propagation in the canine aorta. Circ Res 32:524-529.

[6] Khir AW, O'Brien A, Gibbs JS, and Parker KH (2001) Determination of wave speed and wave separation in the arteries. J Biomech 34:1145-1155.

[7] Laxminarayan S (1979) The calculation of forward and backward waves in the arterial system. Med Biol Eng Comput 17:130-130.

[8] Li JK (1986) Time domain resolution of forward and reflected waves in the aorta. IEEE Trans Biomed Eng 33:783-785. 
[9] Mynard J, Penny DJ, and Smolich JJ (2008) Wave intensity amplification and attenuation in non-linear flow: Implications for the calculation of local reflection coefficients. J Biomech 41:3314-3321.

[10] Mynard JP, Davidson MR, Penny DJ, and Smolich JJ (2010) A numerical model of neonatal pulmonary atresia with intact ventricular septum and RV-dependent coronary flow. Int J Num Meth Biomed Eng 26:843-861.

[11] Mynard JP and Nithiarasu P (2008) A 1D arterial blood flow model incorporating ventricular pressure, aortic valve and regional coronary flow using the locally conservative Galerkin (LCG) method. Comm Num Meth Eng 24:367-417.

[12] Parker KH and Jones CJ (1990) Forward and backward running waves in the arteries: analysis using the method of characteristics. J Biomech Eng 112:322-326.

[13] Pythoud F, Stergiopulos N, Bertram CD, and Meister JJ (1996) Effects of friction and nonlinearities on the separation of arterial waves into their forward and backward components. J Biomech 29:1419-1423.

[14] Pythoud F, Stergiopulos N, and Meister JJ (1995) Forward and backward waves in the arterial system: nonlinear separation using Riemann invariants. Technol Health Care $3: 201-207$.

[15] Pythoud F, Stergiopulos N, and Meister JJ (1996) Separation of arterial pressure waves into their forward and backward running components. J Biomech Eng 118:295301.

[16] Ramsey MW and Sugawara M (1997) Arterial wave intensity and ventriculoarterial interaction. Heart Vessels Suppl:128-134.

[17] Sherwin SJ, Formaggia L, Peiró J, and Franke V (2003) Computational modelling of 1D blood flow with variable mechanical properties and its application to the 
simulation of wave propagation in the human arterial system. Int $\mathbf{J}$ Num Meth Fluids 43:673-700.

[18] Smolich JJ, Mynard JP, and Penny DJ (2011) Pulmonary trunk, ductus arteriosus, and pulmonary arterial phasic blood flow interactions during systole and diastole in the fetus. J Appl Physiol 110:1362-1373.

[19] Smolich JJ, Penny DJ, and Mynard JP (2012) Enhanced central and conduit pulmonary arterial reservoir function offsets reduced ductal systolic outflow during constriction of the fetal ductus arteriosus. Am J Physiol Regul Integr Comp Physiol 302:R175-183.

[20] Stergiopulos N, Tardy Y, and Meister JJ (1993) Nonlinear separation of forward and backward running waves in elastic conduits. J Biomech 26:201-209.

[21] Westerhof N, Sipkema P, van den Bos GC, and Elzinga G (1972) Forward and backward waves in the arterial system. Cardiovasc Res 6:648-656. 
Fig. 1. (a) Single segment model of length $l$, with a forward component of pressure $\left(p_{+}\right)$at the inlet and prescribed pressure reflection coefficient $\left(\Gamma_{p}\right)$ at the outlet. (b) The pressurearea and (c) pressure-wave speed relations for $b=0.1,2.8$ and 8.0. Note: pressures are plotted relative to the reference pressure, $p_{0}$.

(a)

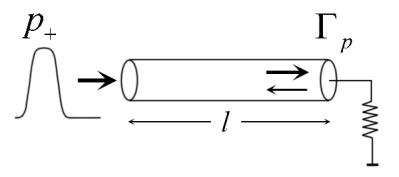

(b)

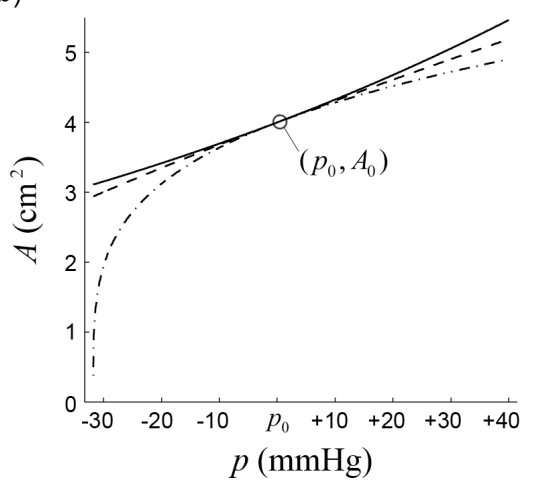

(c)

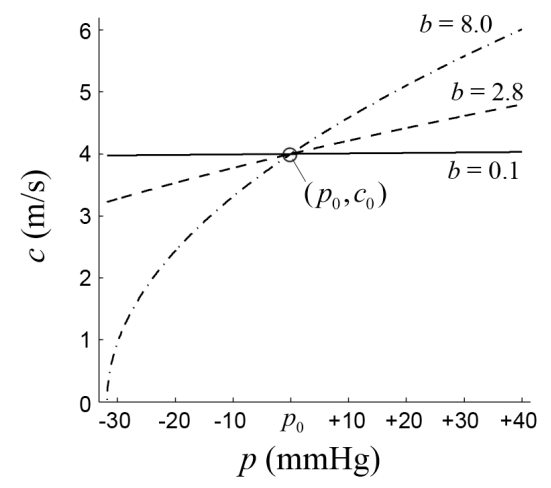


Fig. 2. Net wave speed $(c)$, pressure $(p)$, velocity $(u)$ and wave intensity $(w i)$ and the associated forward and backward components using the linear or non-linear separation (thick dashed or thin solid lines respectively) and for linear, moderately non-linear and substantially non-linear flow conditions $(b=0.1,2.8$ and 8.0 respectively). Percentage linear separation errors for $p_{ \pm}$and $u_{ \pm}$are calculated as the instantaneous difference between linear and nonlinear signals divided by the amplitude of the non-linear signal. Horizontal lines demarcate wi peaks.

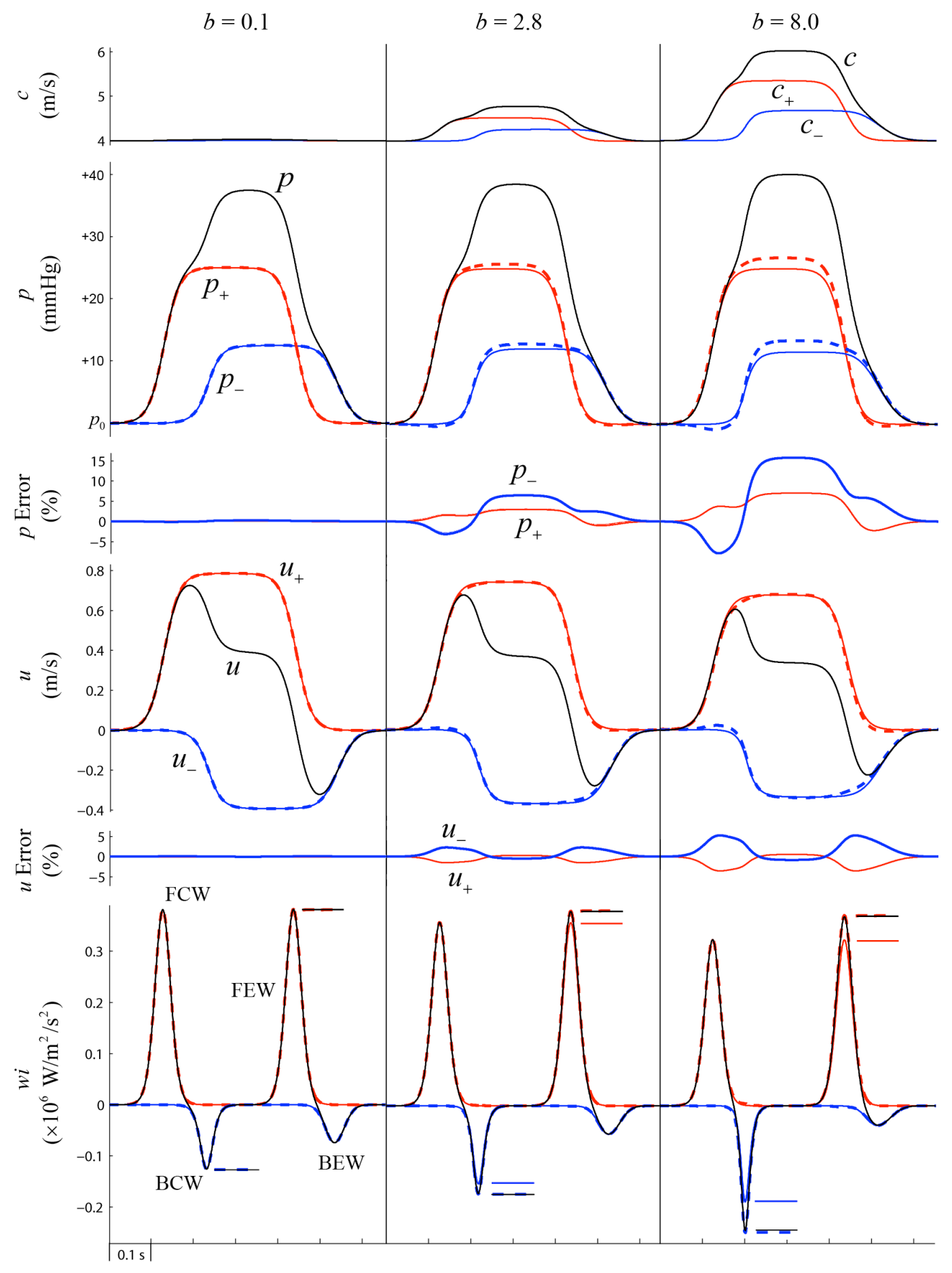


Fig. 3. As in Fig. 2, but with the mean, minimum or maximum wave speed used for calculating the linear separation $(b=8)$. Arrows indicate artefactual backward waves with appreciable pressure/velocity effects.
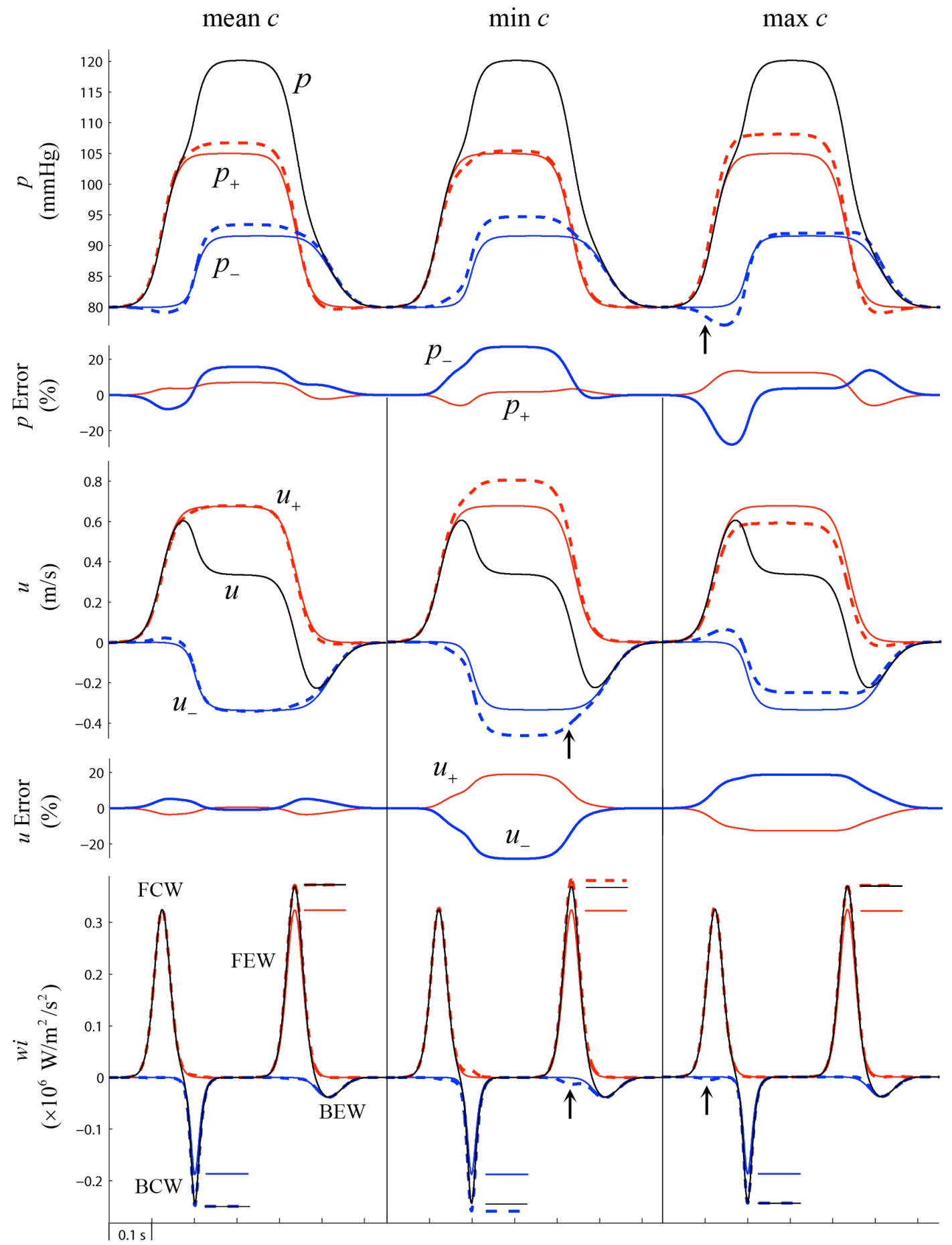
Fig. 4. Maximum linear separation errors for (a) pressure, (b) velocity, and (c) wave intensity, the latter with respect to the backward compression $(\mathrm{BCW})$ and forward expansion (FEW) waves. Parameters tested are wave speed (left panels), terminal reflection coefficient (centre panels) and input pulse amplitude (right panels), for $b=2.8$ (filled symbols) and $b=8.0$ (open symbols). Note that the ' $\mathrm{BCW}$ ' is actually a $\mathrm{BEW}$ when the reflection coefficient is negative.

(a)

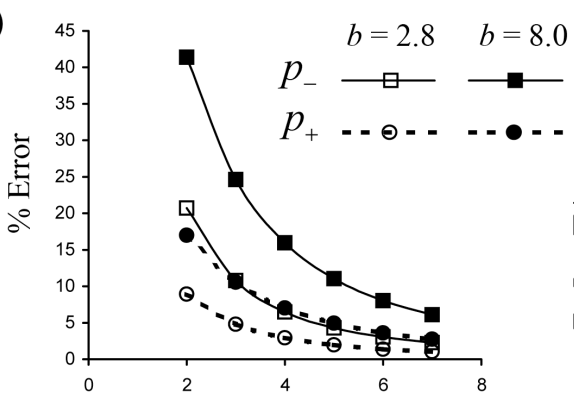

(b)

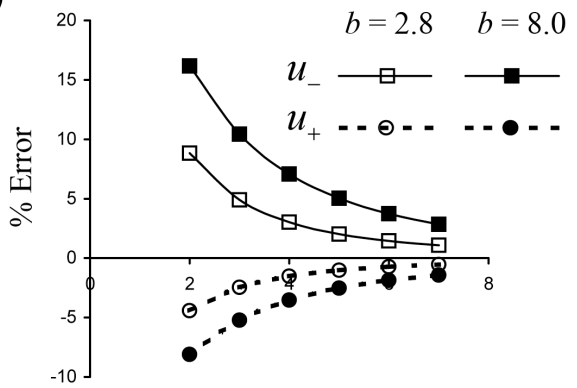

(c)

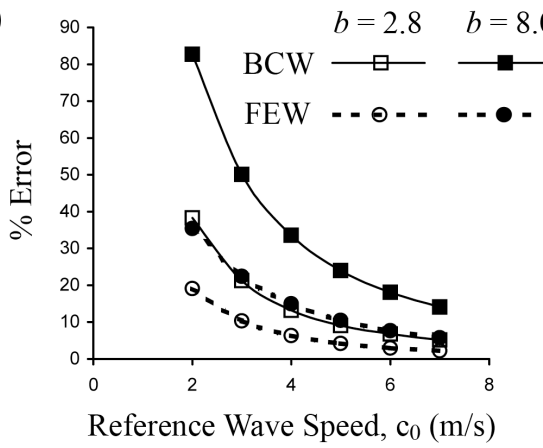

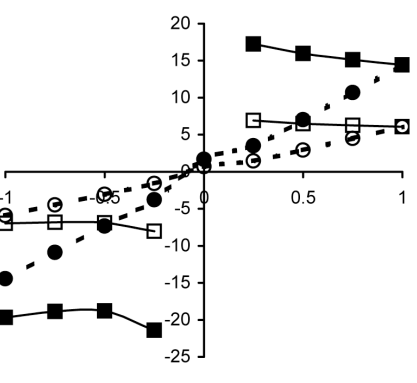
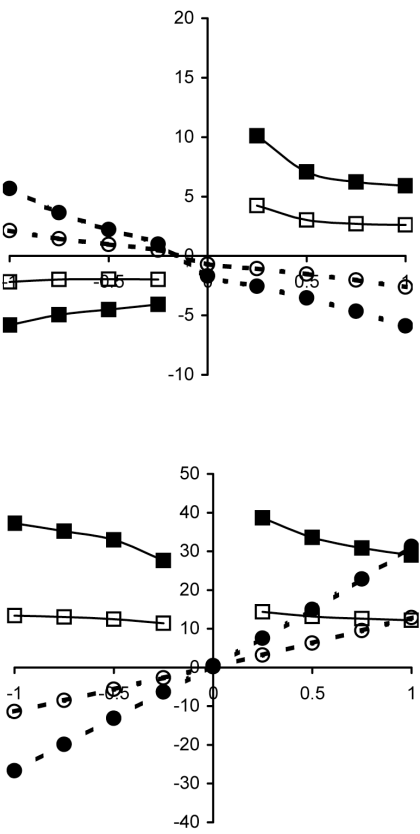

Reflection Coefficient
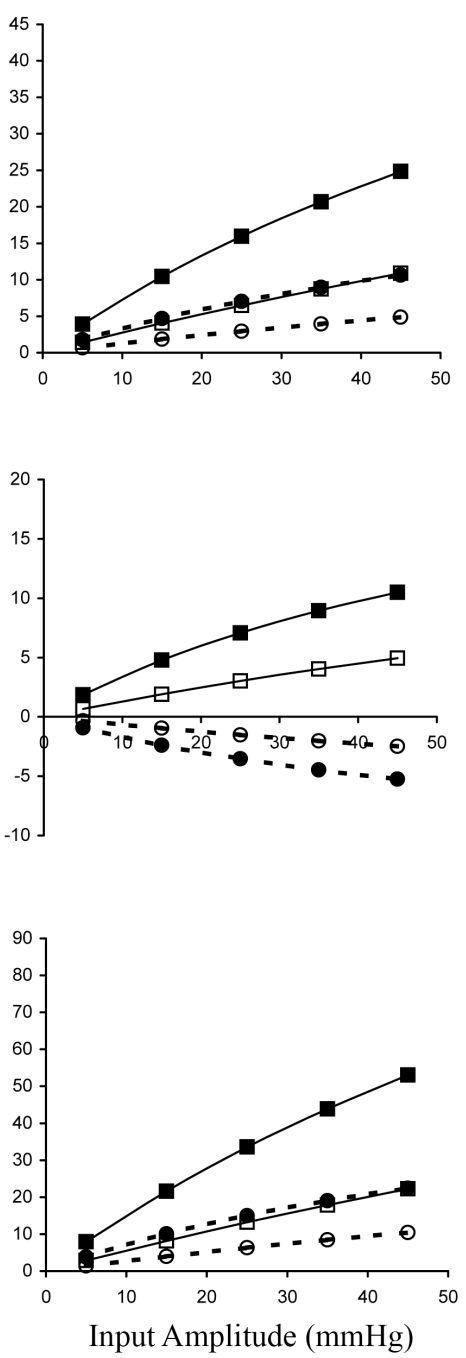


\section{University Library}

\section{- M M N E R VA A gateway to Melbourne's research publications}

Minerva Access is the Institutional Repository of The University of Melbourne

Author/s:

Mynard, JP;Davidson, MR;Penny, DJ;Smolich, JJ

Title:

Non-linear separation of pressure, velocity and wave intensity into forward and backward components

Date:

2012-06-01

Citation:

Mynard, J. P., Davidson, M. R., Penny, D. J. \& Smolich, J. J. (2012). Non-linear separation of pressure, velocity and wave intensity into forward and backward components. MEDICAL \& BIOLOGICAL ENGINEERING \& COMPUTING, 50 (6), pp.641-648. https://doi.org/10.1007/ s11517-012-0897-5.

Persistent Link:

http://hdl.handle.net/11343/127496 